\title{
Using global arrays to investigate internal-waves and mixing
}

\author{
Jennifer MacKinnon, ${ }^{1}$ Matthew Alford, ${ }^{2}$ Pascale Bouruet-Aubertot, ${ }^{3}$ Nathan Bindoff, ${ }^{4}$ Shane Elipot, ${ }^{5}$ Sarah Gille, ${ }^{6}$ James \\ Girton, ${ }^{7}$ Mike Gregg, ${ }^{8}$ Robert Hallberg, ${ }^{9}$ Eric Kunze, ${ }^{10}$ Alberto Naveira Garabato, ${ }^{11}$ Helen Phillips, ${ }^{12}$ Rob Pinkel, ${ }^{13}$ Kurt \\ Polzin, ${ }^{14}$ Tom Sanford, ${ }^{15}$ Harper Simmons, ${ }^{16}$ Kevin Speer ${ }^{17}$ \\ 1 Corresponding author, Scripps Institution of Oceanography, USA, jmackinn@ ucsd.edu \\ 2 University of Washington, USA, malford@apl.washington.edu \\ 3 LOCEAN/IPSL, France, Pascale.Bouruet-Aubertot@locean-ipsl.upmc.fr \\ 4 University of Tasmania, Australia,n.bindoff@utas.edu.au \\ 5 Proudman Oceanographic Laboratory,UK, ship@pol.ac.uk \\ 6 Scripps Institution of Oceanography,USA, sgille@ucsd.edu \\ 7 University of Washington, USA, girton@apl.washington.edu \\ 8 University of Washington, USA, gregg@apl.washington.edu \\ 9 Geophysical Fluid Dynamics Laboratory,USA, Robert.Hallberg@noaa.gov \\ 10 University of Victoria, Canada, kunze@uvic.ca \\ 11 National Oceanographic Center,UK,acng@noc.soton.ac.uk \\ 12 University of Tasmania, Australia, h.e.phillips@utas.edu.au \\ 13 Scripps Institution of Oceanography,USA, rpinkel@ucsd.edu \\ 14 Woods Hole Oceanographic Institution,USA, kpolzin@whoi.edu \\ 15 University of Washington, USA, sanford@apl.washington.edu \\ 16 University of Alaska,USA, hsimmons@sfos.uaf.edu \\ 17 Florida State University, USA, kspeer@ocean.fsu.edu
}

\begin{abstract}
Turbulent diapycnal mixing in the ocean controls the transport of heat, freshwater, dissolved gases, nutrients, and pollutants. Though many present generation climate models represent turbulent mixing with a simplistic diffusivity below the surface mixed layer, the last two decades of ocean mixing research have instead revealed dramatic spatial and temporal heterogeneity in ocean mixing. Climate models that do not appropriately represent the turbulent fluxes of heat, momentum, and $\mathrm{CO}_{2}$ across critical interfaces will not accurately represent the ocean's role in present or future climate. An accurate picture of the worldwide geography of mixing requires a vastly increased database of observations. Unfortunately, traditional microstructure estimates of turbulent mixing are expensive, difficult, and rare. A key development of the last decade has been the development of tools to estimate the turbulent mixing rate from finescale (order 10-50 meter resolution) measurements of internal-wave shear and vertical strain. Global arrays such as the Argo program provide an unprecedented and as yet underdeveloped opportunity to define the global internal wave climate, and in turn identify mixing patterns and hotspots.
\end{abstract}

\section{INTRODUCTION}

The clearest conclusion to emerge from the last several decades of ocean mixing research has been the dramatic patchiness of turbulence in the ocean. For example, Fig. 1 shows depth-averaged turbulent dissipation rate as estimated from fine-scale shear/strain (Section 2.2 below) from the World Ocean Circulation Experiment data. Both the dissipation rate (left panel) and diffusivity (right panel) vary by several orders of magnitude across any given ocean basin. Two patterns visible in this figure are emblematic of global mixing patterns, namely 1) mixing is elevated over rough topography (e.g. where the line crosses the SW Indian Ridge) and 2) turbulent diffusivity often increases with depth.

\subsection{The importance of patchy mixing}

Observing and understanding the global geography of turbulent mixing is crucial for several reasons. First, with- out adequate global sampling we cannot accurately know the average turbulent diffusivity or buoyancy flux at any given depth, or basin-wide. One of the driving questions in small-scale physical oceanography over the last decades has been the perceived order of magnitude difference between the $10^{-4} \mathrm{~m}^{2} \mathrm{~s}^{-1}$ average diffusivity required to power the meridional overturning circulation [3] and the $10^{-5} \mathrm{~m}^{2} \mathrm{~s}^{-1}$ diffusivity most often observed [4]. Several possible resolutions to this discrepancy have been proposed, ranging from enhanced mixing over rough topography $[5,6]$ to wind-driven isopycnal fluxes in the Southern Ocean [7]. However, without significantly improved sampling density we cannot compute accurate global averages of turbulent diffusivity.

Second, the details of mixing distribution can have severe consequences for modeled global circulation. Below the surface mixed layer, most current generation climate models employ a combination of a simple Richardson number parameterization for diffusivity and a horizontally uni- 


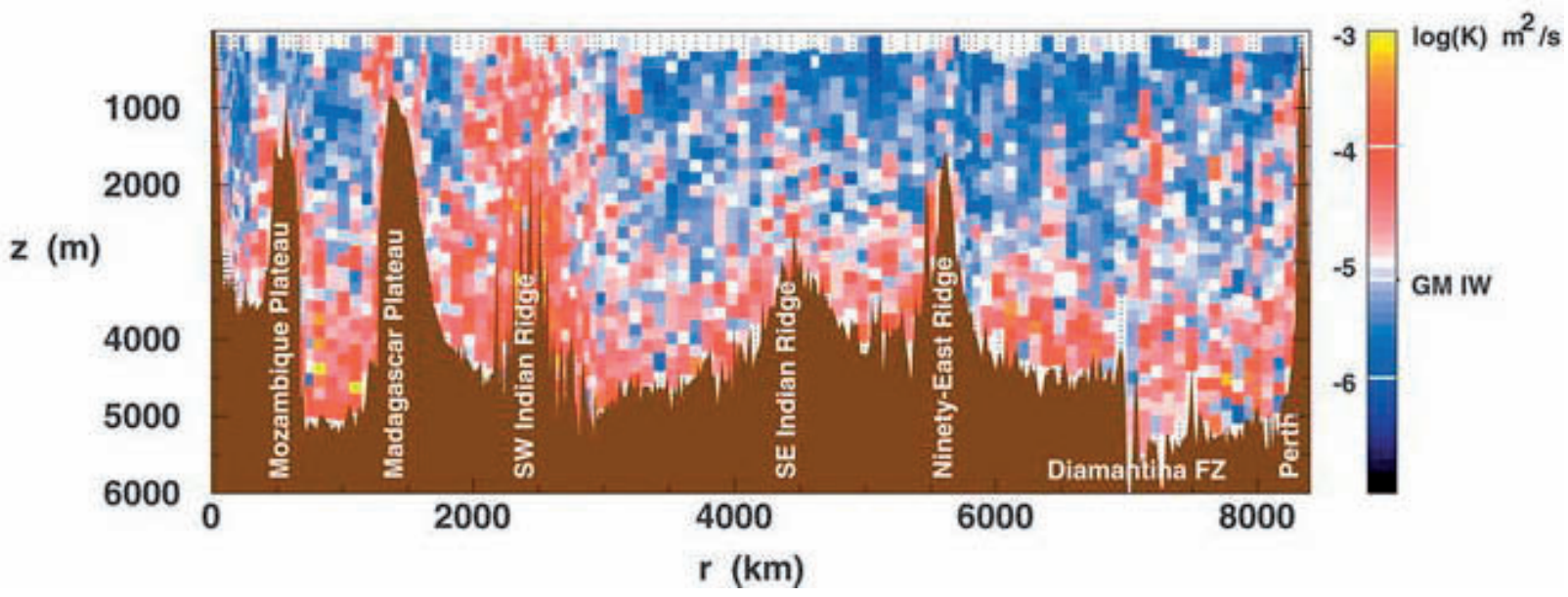

Figure 1: A typical example of vertical and lateral inhomogeneity of diapycnal diffusivity in the ocean: here diffusivity is inferred from WOCE Lowered ADCP data using a finescale parameterization along $32 S$ in the Indian Ocean. White corresponds roughly to the diffusivity expected from wave breaking in a 'background' Garrett-Munk level wavefield, as indicated on the colorbar. Fig. reproduced from $[1,2]$.

form background diffusivity profile. The former are calibrated to (and necessary for) reasonable representation of large-scale sheared flows such as the equatorial undercurrent or high-latitude dense water overflows [8]. However, they are only relevant for cases where the resolved Richardson number leads to turbulent mixing. Climate models do not resolve the vertical scales of breaking internal waves (meters) and will not do so for the forseeable future, so the present suite of Richardson number based parameterizations do not represent the largest source of diapynal mixing in the open ocean. The addition of background diffusivity profiles that generally increase with depth [9] is meant to crudely replicate the zonally averaged structure of turbulent mixing based on observations $[1,10]$. While better than a constant background diffusivity, simple diffusivity profiles ignore the observed horizontal patchiness of mixing, which can have severe consequences for global circulation patterns.

The nature of these consequences depend on the depth (or isopycnal) of diapycnal fluxes. Deep mixing controls the heat and carbon dioxide storage in the ocean and is important for evolution on long timescales. To understand the effect of horizontally patchy mixing, consider the conceptual model of abyssal circulation given by Stommel and Arons. In the traditional view, uniform deep mixing provides a convergent downward turbulent buoyancy flux, with the upwelling rate across any given isopyncal surface roughly given by

$$
A \times w_{*}=\frac{1}{N^{2}} \frac{\partial}{\partial z}\left[\kappa N^{2} A\right]
$$

where $A$ is the area of that isopycnal surface, $N$ the buoyancy frequency, and $\kappa(x, y, z)$ the diapycnal diffusivity. When the diapycnal diffusivity $(\kappa)$ is assumed constant, the positive buoyancy frequency gradient leads to convergent buoyancy flux and upwelling, vortex stretching, and poleward flow. However, when diffusivity is bottom enhanced (Fig. 1), the bracketed term in (1) can be negative, producing local downwelling [11]. While the isopycnal area term, $A$, usually contributes in such a way that there is still net upwelling across each isopycnal surface, the pattern of abyssal circulation can change dramatically if $\kappa$ is laterally inhomogeneous [11-13].

The geography of upper ocean mixing also has a significant impact on oceanic circulation, water properties and heat fluxes. For example, one of the most robust conclusions from two decades of research is that upper ocean mixing has a strong latitudinal dependence, with a steady decline towards the equator, due indirectly to the changing bandwidth of the the internal wave frequency spectrum with changing latitude $[14,15]$. This pattern of upper ocean and thermocline mixing was recently implemented in a global coupled model [16]. They found significantly reduced model biases, changes in equatorial stratification, and reduced heat uptake by the atmosphere compared to model runs with laterally uniform diffusivity. Observations also show episodically enhanced internal-wave driven mixing in the highly stratified transition zone beneath the surface mixed layer [17] that are not represented in current mixed-layer parameterizations. Turbulent fluxes in this region are crucial for mediating air-seat heat exchange and biologically essential nutrient transport.

\subsection{Modeling patchy mixing}

Though diapycnal mixing is only one of many important processes crudely represented by climate models (e.g., cloud physics, oceanic and atmospheric convection, effects of sea-ice heterogeneity, etc.), it is noteworthy for two rea- 
sons. First, as a community we are close to implementing improved parameterizations based on existing theories of wave breaking and (limited) mixing observations. The new observations of wave dynamics and mixing distribution that could be achieved with global arrays would be an enormous help towards getting realistic mixing schemes in IPCC-class models over the next decade. Second, unlike other processes, the problem of diapycnal mixing will not be solved by anticipated increasing model resolution. For example, at the moment lateral/isopycnal mixing due to eddy stirring is also parameterized in climate models with a variety of more or less problematic methods. Fortunately, over the next decade climate models will increasingly become eddy-resolving, in some sense solving this problem. However, diapycnal mixing is driven by turbulent eddies on the scales of $\mathrm{cm}$ to $\mathrm{m}$ (horizontally as well as vertically), which will never be resolved in climate models. Thus the processes that produce diapycnal mixing will always need to be parameterized, and new data will greatly help development of proper parameterizations.

A few global models are beginning to implement simple patchy mixing parameterizations like that described in [13]. Ultimately, however, we need to develop a dynamic parameterization of turbulent mixing for inclusion in climate models that will allow the magnitude and distribution of mixing to evolve in a changing climate. Wunsch and Ferrari sum up the importance of understanding mixing patterns for climate models [18]:

A number of lines of evidence, none complete, suggest that the oceanic general circulation, far from being a heat engine, is almost wholly governed by the forcing of the wind field and secondarily by deep water tides... The now inescapable conclusion that, over most of the ocean, significant vertical mixing is confined to topographically complex boundary areas implies a potentially radically different interior circulation than is possible with uniform mixing. Whether ocean circulation models... neither explicitly accounting for the energy input into the system nor providing for spatial variability in the mixing, have any physical relevance under changed climate conditions is at issue.

\subsection{Dynamics that drive mixing}

Away from the direct influence of boundary processes, most ocean mixing is driven by breaking internal gravity waves. Energy is input into the internal wave field primarily by the tides and wind [18]. Internal tides are generated where the barotropic tide rubs over rough topography. Some of the resultant baroclinic energy dissipates locally, producing a global map of mixing hotspots that mirrors internal tide generation sites [6]. However, most of the energy radiates away in the form of low (vertical) mode waves [19]. Where this low-mode energy dissipates is still very much up in the air - contenders include scattering over deep topography, breaking on the continental slope, or nonlinear interactions with the ambient internal wave field (including the special case of parametric subharmonic instability), among others.

Near-inertial internal waves start with surface wind forcing of near-inertial motions in the mixed layer [20]. Beta-plane and eddy-interactions change the horizontal wavenumber so this variance can move equatorward and into the pycnocline, turning purely inertial motions into near-inertial waves that can propagate $[21,22]$. Subsequent interactions within the internal wave field and with topography likely determine their role in turbulence production but these pieces of the puzzle are not well-understood. Local dissipation of higher-mode near-inertial waves plays a large role in turbulent fluxes of heat, dissolved gases, and nutrients in the stratified transition layer just beneath the mixed layer. As with internal tides, higher-mode waves are likely to be generated and dissipated locally, while lowmode waves escape to propagate thousands of $\mathrm{km}$ across ocean basins [23].

Before a dynamically based parameterization of mixing can be developed for climate models, questions like the following need to be addressed:

1. How far (vertically and horizontally) does elevated mixing over rough topography extend?

2. What controls mixing in the transition layer (beneath the surface mixed layer). How does this mixing correlate with wind stress and surface buoyancy fluxes?

3. How do internal-waves interact with mesoscale shear or vorticity to dissipate energy? We still know very little about how geostrophic flows give up their energy, and progress requires a better understanding of relationships between the eddy and internal wave fields.

Answers to these questions require significantly more knowledge on small-scale processes from a variety of environments. Such knowledge can be obtained by surveying both continuously and extensively for a number of years, as could be done with a combination of higherresolution Argo data, surface altimetry, and the addition of velocity profiles

The goal of this white paper is twofold: 1) to describe the current (underutilized) capability of existing global measurement arrays for inferring small-scale dynamics, and 2) advocate additions for the next generation of ocean observing capability that would significantly improve our ability to measure mixing-related properties worldwide. We begin with a brief description on the dynamics of internalwave driven turbulence in the ocean and methods of measuring or inferring the associated mixing rates.

\section{METHODS FOR ESTIMATING MIXING}

Microstructure measurements provide the gold standard for determining diapycnal mixing in the ocean. However, the 
instruments needed to carry out these measurements are costly, and experienced teams are required to deploy and recover them. As a result, microstructure measurements have been conducted in only a limited number of geographic locations across the globe. While it is essential that the community continue to make these measurements, a variety of complementary methods have emerged to approximate vertical diffusivities by taking advantage of finestructure measured by instruments that are more readily available.

\subsection{Turbulent overturns}

The most direct way to measure turbulent mixing is to measure the scales at which turbulent overturns are actually occurring. Turbulent diffusivity $\kappa$ is related to the turbulent dissipation rate $\epsilon$, which is easier to measure, through an assumed mixing efficiency

$$
\kappa \sim \Gamma \frac{\epsilon}{N^{2}},
$$

where $\Gamma$ is typically taken to be $0.2[25,26]$. Since most oceanographic measurements are in the form of vertical profiles, the dimensions of turbulence are often discussed in terms of vertical scales (Fig. 2). Turbulence in stratified water is bounded at the upper end by the Ozmidov scale,

$$
L_{0}=\frac{2 \pi}{m_{0}}=\sqrt{\frac{\epsilon}{N^{3}}} \sim \mathcal{O}(0.1-10 \mathrm{~m})
$$

and at the lower end by the Kolmogorov scale (order of $\mathrm{mm}$ ). The latter is the scale at which viscosity actually diffuses momentum, while the former is related to outer scales of turbulent overturns. Traditional microstructure instruments measure well into the inertial subrange between these scales, and estimates of the turbulent dissipation rate are based on fits to assumed spectra of velocity or temperature fluctuations. This is an accurate but difficult and expensive measurement to make. However, the outer scales of turbulent overturns can often be measured with standard CTD sensors, provided that the data are saved at a high enough resolution. The quantity typically calculated is known as the Thorpe scale $\left(L_{t}\right)$, defined as the root mean squared displacement a parcel has moved between a measured density profile with a density inversion (overturn) and the sorted version of the same profile. The Thorpe scale has been shown to be a good estimate of the Ozmidov scale $\left(L_{t} \sim L_{0}\right)$, so CTD measurements of density inversions can be used to estimate $\epsilon$ through (3) [27-29]. The results generally compare well with microstructure estimates (e.g. [29-31], Fig. 3). The largest drawback of this method is the accuracy required to measure density overturns at scales of meters or less. For low-level turbulence and weak stratification these scales are right up against the noise level of most CTDs. However, in energetic regimes turbulence may produce overturns of tens of meters or more, which should be detectable by a wide variety of platforms.

\subsection{Finescale parameterizations of mixing}

Over the last few decades, a variety of empirical, statistical, and theoretical models have been developed to relate finescale (tens to hundreds of meters vertically) shear and strain from internal waves to the associated turbulent dissipation rate and diffusivity. The basic idea is that weakly nonlinear interactions between a well-developed sea of internal waves act to steadily transport energy from the large (vertical) scales at which it is generated and propagates, to the small scales at which waves break due to shear or convective instabilities. The more energetic the wavefield, the faster this rate of down-scale transfer and the larger the dissipation rate and associated diffusivity [33,34]. More specifically, internal wave shear and strain tend to have flat vertical wavenumber spectra at scales larger than about 10 $\mathrm{m}\left(m_{k}\right.$ in the left panel of Fig. 2). The rate of downscale energy transfer through these scales (and thus the dissipation rate) tends to scale quadratically with the spectral level $\hat{E}$,

$$
\epsilon \sim \hat{E}^{2}
$$

a scaling consistent between theory [14], observations [15, 24,35] (Fig. 2), and numerical simulations [36].

The ability to infer turbulent dissipation rates from finescale quantities has profound implications for developing global estimates of mixing rate. Wijesekera et al. state the case well [37]:

...If [predicting the dissipation rate based on in-
ternal wave dynamics] is even approximately true,
the significant oceanographic problem of estimating
vertical viscosities and diffusivities for large-scale
modeling applications shifts from adequately sam-
pling the processes responsible for mixing to defin-
ing the global internal wave climate.

The Gregg-Henyey-Polzin method, as it is sometimes known, does not work in all environments. In particular, it assumes dissipation is produced by weakly nonlinear interactions in a broadband field of internal waves. The scaling fails where internal waves are strongly nonlinear (e.g. solitons), in shallow water where the internal wave field has a much reduced vertical bandwidth $[38,39]$, or where largescale baroclinic motions are directly breaking. For example, [31] find the G-H-P scaling to be over an order of magnitude too small right above the Hawaiian Ridge, where a large-amplitude internal tide is directly breaking, but agree quite well with microstructure estimates only hundreds of meters away (Fig. 3). Even in the best environments, uncertainties remain as to the error bars associated with this technique. Ongoing detailed comparison between microstructure and finescale parameterizations by a number of investigators should help. 

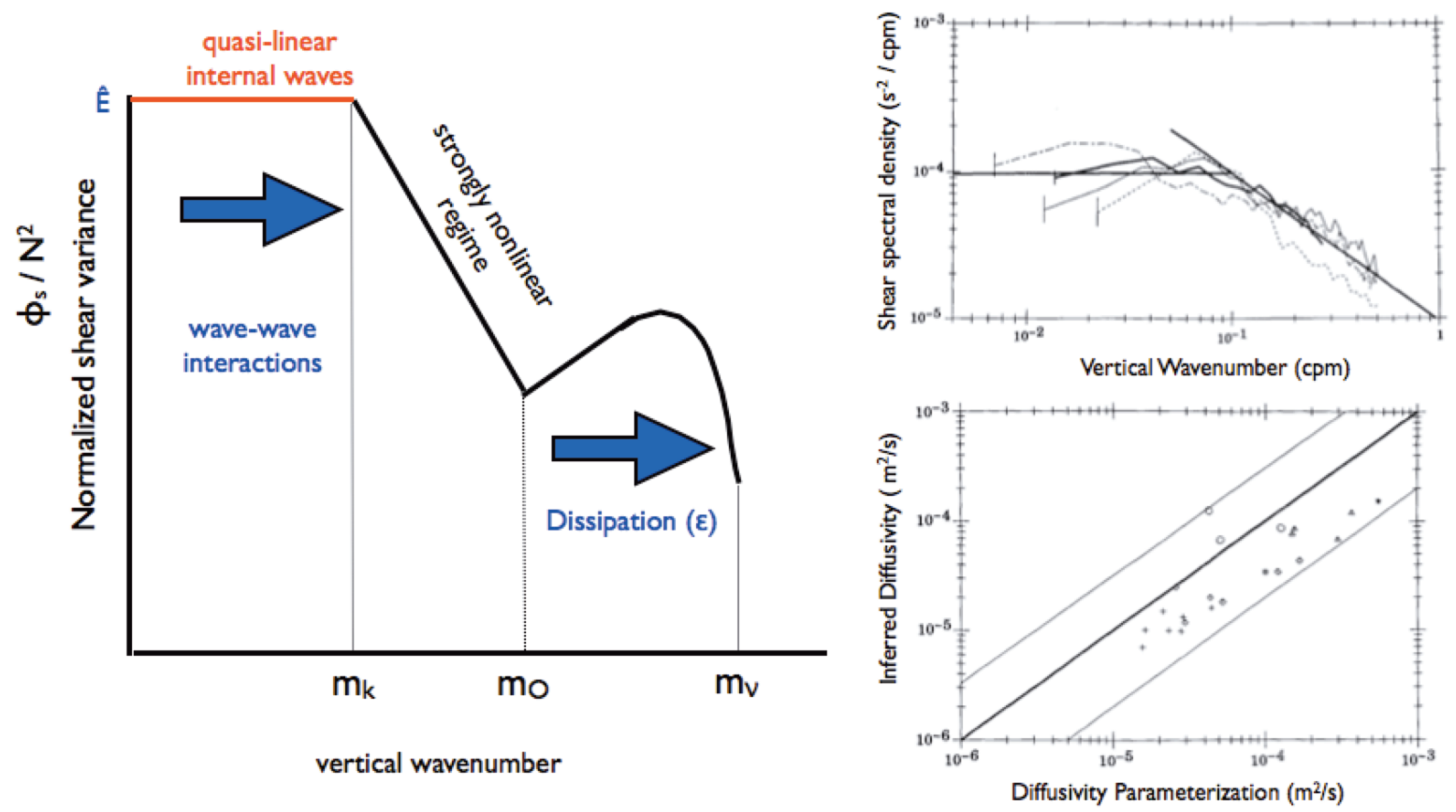

Figure 2: Left: Sketch of idealized vertical wavenumber spectra of stratification normalized shear showing steady-state spectral shapes for the internal wave regime (low wavenumbers / large vertical scales), the transition range, and the turbulent subrange at high wavenumbers / small vertical scales. Wavenumbers indicated on the $x$-axis correspond to the Kolmogorov scale $\left(m_{\nu}\right)$, the Ozmidov scale $\left(m_{O}\right)$, and the edge of the quasi-linear internal wave regime $\left(m_{k}\right)$. The blue arrows schematically indicate the direction of energy transfer from large to dissipative scales. Right: Demonstration of shear spectral shape and Gregg-Henyey-Polzin dissipation rate scaling, from [24]: Shear spectra scaled in the vertical by $1 / E_{1} N^{2}$ and in the horizontal by $E_{1}$ for a variety of data sets (top); diffusivities inferred from microstructure measurements plotted against a fine-scale parameterization showing good agreement (bottom).

\section{ESTIMATING MIXING FROM GLOBAL AR- RAYS}

Global arrays such as the Argo program provide an unprecedented opportunity to improve our knowledge of finescale processes in the ocean. The sheer quantity of data and global coverage represent a huge addition to ship-based measurements. Perhaps their greatest potential is in sampling small-scale processes in inaccessible or inhospitable locations and times, such as the Southern Ocean or beneath winter storms. Such environments are challenging for shipbased observations, yet are likely to be hotspots of turbulent mixing. Below we discuss the potential for measurement of small-scale process from existing instruments and argue for improvements in the next generation of measurements. This is not intended to be a comprehensive list of available data or analysis possibilities, but rather a starting point for ongoing discussion.

\subsection{Using existing measurements}

Applying finescale parameterizations of turbulent dissipation to current global ocean observing system data can dramatically improve global estimates of mixing. The turbu- lent dissipation rate may be estimated by measuring either shear or strain from internal waves at these scales [37], although the most accurate estimates require a combination of the two [1]. In addition to the study pictured in Fig. 1, several other investigators have already applied this method to Lowered ADCP data [40-43]. The strong and bottomenhanced diffusivity calculated by [44] using this method near Drake Passage was a pivotal motivation for the ongoing multinational DIMES (Diapycnal and Isopycnal Mixing Experiment in the Southern Ocean) project. On the other hand, significant uncertainties remain, and care must be taken when interpreting method parameters and sensor noise.

Argo: Standard Argo floats make use of the ARGOS transmission system and are able to transmit a limited number of data points per profile. Typically they attain vertical resolutions of 5 to $10 \mathrm{~m}$ in the upper ocean and $50 \mathrm{~m}$ in the deep ocean. In most cases, this vertical resolution is unlikely to be sufficient to infer any information about finestructure mixing. However, Argo floats that make use of the Iridium communications system are able to send more data, and some already provide profiles that may be sufficient for finestructure calculations. Moreover, if the Argo array is transitioned to make use of Iridium (or any other 

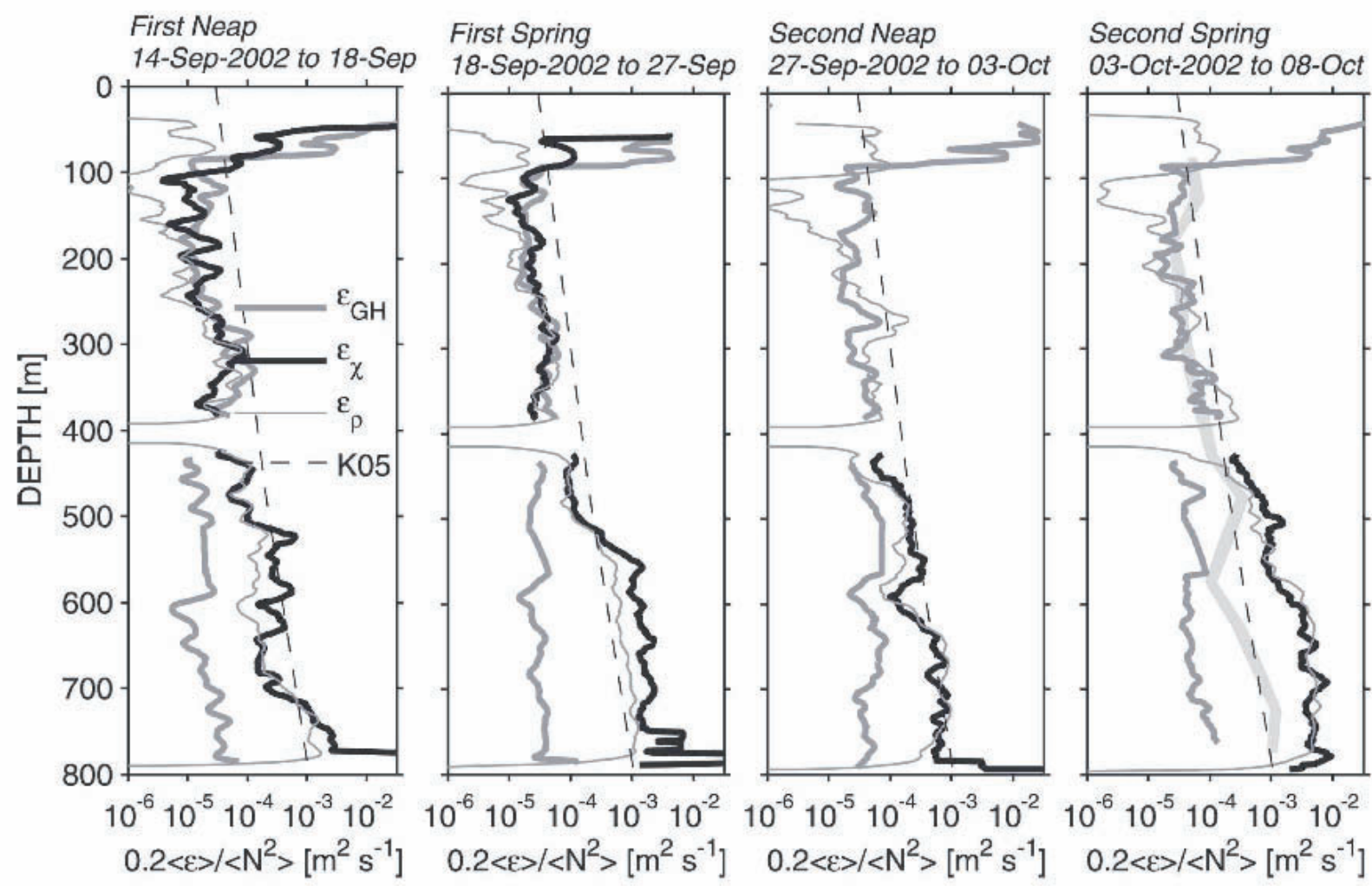

Figure 3: A comparison of several methods for calculating the turbulent dissipation rate, $\epsilon$, normalized by mean stratification (to make a diapycnal diffusivity) for four time periods near Oahu. Data were taken during the Hawaiian Ocean Mixing Experiment and are reproduced from [31]. In each panel the thick black line is the estimate from the microconductivity probe; thin shaded line is from density overturns; thick shaded line is from GreggHenyey parameterization. There was no microconductivity from the upper CTD for the second two time periods. The dashed line is the composite dissipation profile from direct turbulence measurements made in deep water atop the ridge [32].

satellite communication system that allows higher volumes of data to be sent) then higher vertical resolution could be achieved for the entire array. Though CTD data can be contaminated by ship roll, Argo floats rise freely through the water column and are unaffected by this. Thus they have the potential to provide high-quality profiles well suited for finestructure calculations. There is no clear cutoff to indicate what vertical resolution must be achieved to make Argo floats useful for finestructure calculations, but we expect that for most regions with typical background stratification, 1 to $2 \mathrm{~m}$ resolution data would be usable.

Ship based measurements: In addition to finescale estimates of mixing from lowered ADCP data, Thorpe scales can be calculated from standard shipboard CTDs, provided some care is taken to understand relevant noise levels and the contaminating effect of ship motion $[45,46]$. Thorpe scales can also be calculated from expendable CTDs, which do not have the problem of ship motion. XCTDs are regularly deployed along repeat measurement lines, such as WOCE transect AX22 in the Drake Passage [47].
Mooring arrays: Long-term mooring collections such as the RAPID array involve ADCPs, moored profilers, and thermistor chains that may be used for finescale estimates of mixing. Even where resolution is not high enough to observe breaking waves, long term information on the internal wave climate in the deep ocean is an important part of the global energetics.

\subsection{New tools and measurement strategies}

One of the most straightforward improvements to global measurements would come from consistently archiving high-resolution CTD data. In order for Thorpe scales or fine-scale strain to be calculated from shipboard CTD data, the data must be archived and made available at the highest possible resolution (raw). Standard processing into 1-2 dbar bins is not adequate. Even when features of interest (overturns) are several meters tall or more, careful postprocessing in order to remove ship roll and salinity spikes is required to have the accuracy necessary to confirm density inversions [46]. This processing is only possible if PIs have 

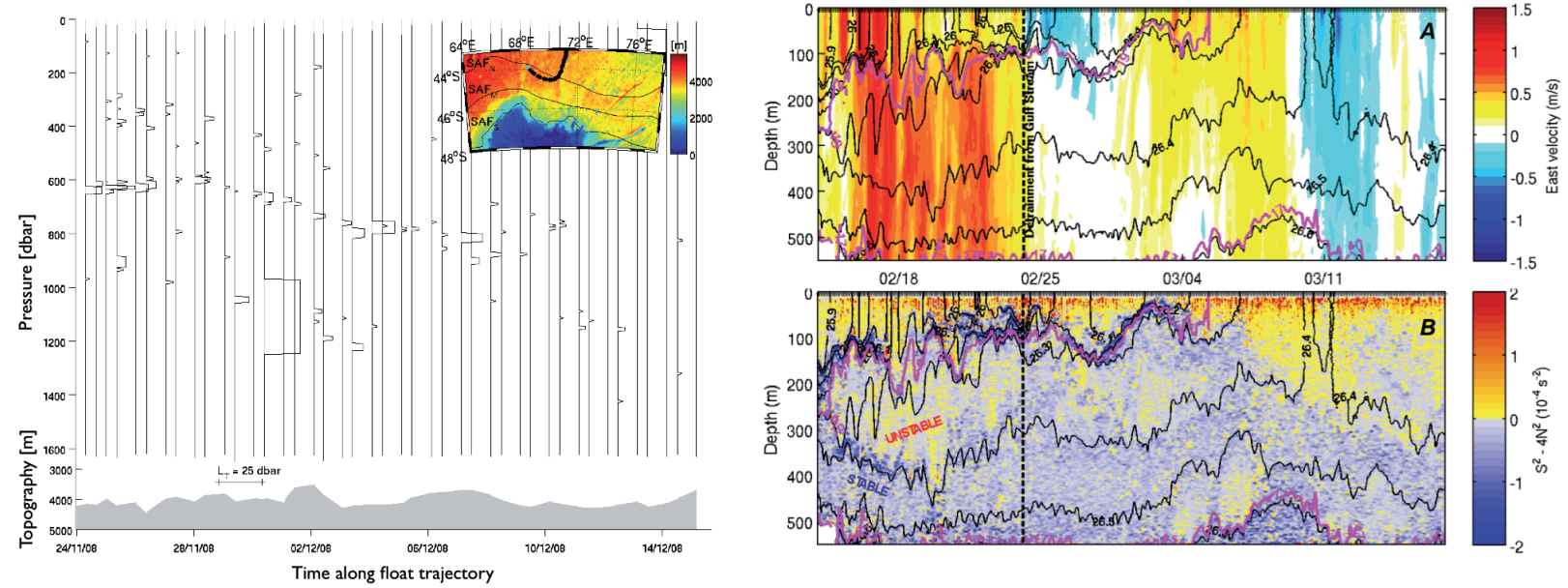

Figure 4: Examples of EM-APEX float measurements. Left: Thorpe scales calculated from an EM-APEX float, vertical resolution approximately 2 dbar, deployed at the northern Kerguelen Plateau (see inset map). Each line corresponds to a float profile and each spike on the profiles matches a turbulent patch where the horizontal length of the spike (in the plot) represents the vertical Thorpe scale for a particular overturning patch, $\mathrm{L}_{\mathrm{t}}$. The inset map also shows the local topography and the position of the northern, middle and southern Subantarctic Front ( $\mathrm{SAF}_{\mathrm{N}}, \mathrm{SAF}_{\mathrm{M}}$, and $\mathrm{SAF}_{\mathrm{S}}$ respectively). [ Unpublished results from Ph.D. research of Amlie Meyer, University of Tasmania, Australia.] Right: (upper) Zonal velocity and (lower) Reduced shear $\left(S^{2}-4 N^{2}\right)$ from an EM-APEX float in the 2007 CLIMODE experiment in the Gulf Stream. The velocity measurement shows both the along-stream velocity evolution and high-frequency variability due to internal waves (primarily storm-driven near-inertial waves). The reduced shear (a quantity that is positive when the Richardson number is below 1/4 and the flow is susceptable to Kelvin-Helmholtz instability) indicates the most likely regions to undergo mixing. Black lines are density contours in $0.1 \mathrm{~kg} / \mathrm{m}^{3}$ intervals, and $17^{\circ}$ and $19^{\circ}$ isotherms are in magenta

access to the raw data.

Global finescale measurements from the Argo array would be significantly enhanced by three primary changes:

- Improved vertical resolution. Higher vertical sampling would not only allow far more accurate estimates of internal wave strain, but in some environments would allow direct observation of turbulent overturns. For example, Meyer and Phillips (Fig. 4, left) are able to calculate Thorpe scales (Sec. 2.1) in the energetic Kerguelan Plateau region. Though they are using EM-APEX floats, similar calculations could be done with any of the Argo array provided the vertical resolution was high enough. Increasing the vertical resolution requires a financial commitment to the bandwidth required to send higher density data. An Argo array with enhanced resolution would also allow us to resolve lateral (isopycnal) stirring processes and the higher modal structures of mesoscale eddies (higher than mode one) that are key to determining and understanding the controls of eddy mixing rates.

- Deeper measurements. Turbulent diffusivity is often bottom enhanced (Fig. 1), especially over rough topography. Extension of the Argo array to include measurements below 2000 meters would make an invaluable improvement towards our understanding of deep mixing in the worlds oceans. We emphasize that mixing is elevated for hundreds of meters over rough topography, so the profile would not need to be close to the bottom to sample more regions of enhanced mixing. Argo floats are calibrated before launch but are not recovered for post-measurement calibration. For climate research this can pose a challenge, particularly in the deep ocean where horizontal temperature gradients are small, since temporal evolution of temperature or salinity may be difficult to distinguish from instrument drift. However, finestructure measurements are not subject to calibration difficulties since they depend only on the vertical gradients measured within a single profile.

- Adding velocity measurements. The new EM-APEX platform (described in more detail below) adds horizontal velocity to the standard Argo package at about a $60 \%$ increase in float cost (which could potentially come down to $20-30 \%$ with sufficient volume). In addition to the many other applications of velocity profiles, the information gained about the internal wave field is a significant improvement over what is possible with CTD (strain) alone. The shear-to-strain ratio of internal waves depends on their frequency and wavelength, as well as the environmental parameters of latitude and stratification, so strain alone can miss important details of internal wave generation as well as the scaling of dissipation. 
EM-APEX floats - capability, strategies, examples.

A new variant of the standard Webb Research APEX profiling float used in Argo is now available with a subsystem for measuring motionally induced electric fields generated by the ocean currents moving through the vertical component of the Earth's magnetic field. Since this is a more significant change than other proposed array enhancements, we include some technical detail here. Electrodes on the upper end cap, below the SBE-41 CTD, sense the motionally induced voltages. The voltages are amplified, digitized, processed into velocity components and stored within the float. Other measurements are components of the Earth's magnetic field (i.e., compass) and instrument tilt. Float position is determined by the global positioning system when the float surfaces. The T, S, V, position, and other observations are processed within the float and transmitted over the Iridium global cell phone system. The Iridium link is bidirectional, allowing not only data uploads but also downloads of mission changes.

The horizontal electric field as observed on a platform moving with the surrounding water is

$$
\nabla_{h} \phi_{a}=-F_{z}\left(\mathbf{v}-\overline{\mathbf{v}}^{*}\right) \times \mathbf{k}-\mathbf{J}^{*} / \sigma,
$$

where $\phi_{a}$ is the apparent potential around a moving sensor (N.B. after correction is applied for the distortion of the electric currents around the APEX float), $F_{z}$ is the vertical component of the Earth's magnetic field, $\sigma$ is electrical conductivity, $\mathbf{v}(z)$ is local water velocity, $\overline{\mathbf{v}}^{*}$ is a vertically integrated, conductivity weighted ocean velocity, and $\mathbf{J} *$ represents non-local electric currents (typically negligible). The important point is that only one term varies with depth. It is this term that provides the vertical distribution of current. The other terms represent an unknown (but knowable with an independent velocity measurement, such as of the surface velocity from GPS observations), depth-independent offset. As the profiler falls/rises at $0.1-0.12 \mathrm{~ms}^{-1}$ and rotates slowly, the electric field is measured every 1-s and fitted over $50 \mathrm{~s}$ to sine and cosine components derived from the magnetic sensor. This yields a velocity value every 5-6 $\mathrm{m}$. The fit is moved $25 \mathrm{~s}$ and repeated. Thus, velocity values are computed every $2.5-3 \mathrm{~m}$ in the vertical. With alkaline batteries, the instrument should provide 150 profiles to 2000 dbar. Lithium batteries will provide many more profiles. EM-APEX equipped with Lithium batteries, deployed north of the Kerguelen Plateau in the SOFINE experiment, have recorded in excess of 300 profiles to $1600 \mathrm{dbar}$ and are still operating.

EM-APEX floats have so far been deployed in focused process experiments, including CBLAST, EDDIES, CLIMODE, PhilEx, SOFINE, and DIMES, and are components of several upcoming experiments, such as ITOP (typhoon) and LatMix ONR projects. Fig. 4 shows an example of velocity and shear measurements from the Gulf Stream (CLIMODE). [48] and [49] used EM-APEX profiles from the first deployment, in Hurricane Frances in 2004, to measure the momentum flux into the ocean (used to derive new values of drag coefficient), SML cooling, and surface gravity wave amplitudes. In addition, the data provided valuable information for numerical modeling of the ocean responses to high wind stress events, such as tropical cyclones.

Novel profiling strategies can significantly help our understanding of the internal wave climate. For example, conducting pairs of vertical profiles separated by half an inertial period allows easy identification of near-inertial shear. Fig. 5 shows subsurface near-inertial energy obtained from 2 months of data from 3 EM-APEX floats in the Southern Ocean. By differencing profiles separated by $1 / 2$ inertial period (about 7 hours at this latitude) a clear picture of the downward propagation of near-inertial energy emerges.

\subsection{Surface drifters and near-inertial oscillations}

The global surface drifter array is an excellent tool for observing near-inertial oscillations in the surface ocean, especially in combination with suggested EM-APEX floats to observe sub-surface evolution into near-inertial internal waves. Due to large spatial and seasonal variability, it is difficult to design ship-based observational programs to observe near-inertial wave generation and evolution. However, drifting global arrays provide an ideal platform, provided that they sample at a high enough temporal resolution to observe near-inertial motions. Until December 2004, the array of surface drifters from the Global Drifter Program was tracked by two Argos satellites resulting in 6 to 9 daily positioning fixes at Equatorial latitudes to a theoretical 28 fixes per at the poles [51]. From January 2005, NOAA negotiated the use of the full Argos satellite constellation (5 to 6 satellites). As a result, we have now achieved 16-20 fixes per day at the equator and more towards higher latitudes. The global average for the time interval between two drifter fixes is now 1.2 hours. As a result, oceanic variability at high frequencies, including near-inertial waves and super-inertial variability, is captured by surface drifter displacement, even in the high latitude ocean [52].

Several groups have already begun to take advantage of this resource for looking at high-frequency processes in the upper ocean. In a recent paper, [50] used surface drifter velocity data to compute a global seasonal climatology of near-inertial currents (Fig. 5) that confirmed and extended the earlier pioneer work of [53] who obtained characteristics of near-inertial motions on large scales based on the surface drift of Argo floats. [50] compared their observations of near-inertial energy to predictions by Pollard and Millard's [1970] slab-layer model of near-inertial motions and found great discrepency with the drifter observations. They therefore questioned the estimates of wind energy input to inertial motions based on this model. This suggests that estimates of wind energy input to inertial motions, potential energy for deep mixing, need to be re-evaluated. [54] find near-inertial oscillations are significantly modified by geostrophic vorticity.

The GDP array currently comprises about 1250 drifters 

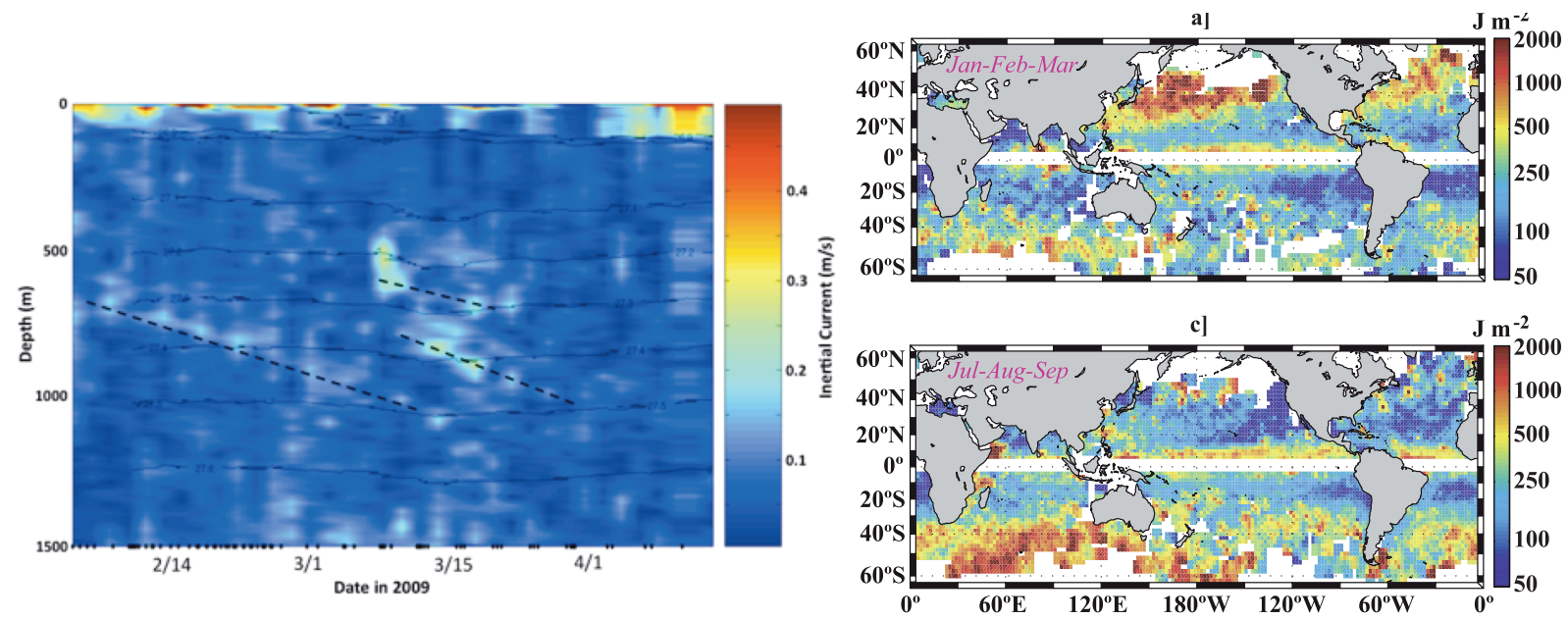

Figure 5: Left: Amplitude of the near-inertial currents vs. depth and time observed by 3 EM-APEX floats in the 2009 DIMES experiment $\left(59^{\circ} \mathrm{S}, 107^{\circ} \mathrm{W}\right)$, showing downward-propagating beams of energy. The near-inertial component is determined by differencing pairs of profiles separated at 1/2 inertial period. Because the most prominent features (dashed lines) are sampled by each of the floats individually (at separations of 10-50 km), the three have been combined into a single timeseries to increase the signal-to-noise ratio. Potential density $\left(\sigma_{\theta}\right)$ contours are overlain in black. Right:Seasonal variation of inertial mixed-layer energy computed from satellite-tracked drifter trajectories over January-March (upper) and July-September (lower), adapted from [50].

that are managed by AOML. A complete understanding of the dynamical implications of near-inertial variability present in this dataset has yet to be accomplished. Nevertheless, it seems crucial to maintain the multi-satellite tracking, as well as the drifter array itself. The drifters by themselves only record the surface signatures of inertial waves. Srifice etpher lepllfporal resolution of drifter displacement has increased, there may be a risk that the position noise level is reached (at least for spectral descriptions of the variability). It will be necessary to understand better the behavior of drifters in high winds and high wave environments. If the new generation of Argo floats allow us to resolve the interior field of internal waves, the linkage between the GDP array and the Argo array could potentially provide a way to monitor the generation and downward propagation processes for near-inertial energy, and ultimately its dissipation as turbulent mixing.

\section{CONCLUSIONS}

Understanding the magnitude and geography of diapycnal mixing remains one of the outstanding challenges of physical oceanography. A better understanding of both current values and the dynamical processes that produce them is required before accurate mixing parameterizations can be implemented in global climate models. Global measurement arrays can be an integral component of an improved understanding. Mixing may be estimated from a variety of fixed and drifting platforms using finescale estimates of turbulent mixing rates. Some of these calculations can be done with existing data. However, significant advances require im- proved vertical resolution, improved depth sampling, and addition of velocity sensors.

\section{References}

1. Kunze, E., Firing, E., Hummon, J. M., Chereskin, T. K. \& Thurnherr, A. M. (2006). Global abyssal mixing inferred from lowered ADCP shear and CTD strain profiles. J. Phys. Oceanogr. 36, 1553-1576.

2. Kunze, E., Firing, E., Hummon, J. M., Chereskin, T. K. \& Thurnherr, A. M. (2006). Corrigendum. J. Phys. Oceanogr. 36, 2350-2353.

3. Munk, W. H. (1966). Abyssal recipes. Deep-Sea Res. 13, 707-730.

4. Gregg, M. Estimation and geography of diapycnal mixing in the stratified ocean. In Imberger, J. (ed.) Physical processes in lakes and oceans, Coastal and Estuarine Studies, 305-338 (American Geophysical Union, 1998).

5. Polzin, K., Toole, J., Ledwell, J. \& Schmitt, R. (1997). Spatial variability of turbulent mixing in the abyssal ocean. Science 276, 93-96.

6. St. Laurent, L., Simmons, H. \& Jayne, S. (2002). Estimating tidally driven mixing in the deep ocean. Geophys. Res. Lett. 29, doi:10.1029/2002GL015633.e.

7. Toggweiler, J. \& Samuels, B. (1998). On the ocean's largescale circulation near the limit of no vertical mixing. J. Phys. Oceanogr. 28, 1832-1852.

8. Jackson, L., Hallberg, R. \& Legg, S. (2008). A parameterization of shear-driven turbulence for ocean climate models. $J$. Phys. Oceanogr. 38, 1033-1053.

9. Bryan, K. \& Lewis, L. (1979). A water mass model of the world ocean. J. Geophys. Res. 84, 2503-2517. 
10. Gregg, M. C. (1977). Variations in the intensity of small-scale mixing in the main thermocline. J. Phys. Oceanogr. 7, 436454.

11. Simmons, H., Jayne, S., Laurent, L. S. \& Weaver, A. (2004). Tidally driven mixing in a numerical model of the ocean general circulation. Ocean Modelling 6, 245-263.

12. Saenko, O. \& Merryfield, W. (2005). On the effect of topographically enhanced mixing on the global ocean circulation. J. Phys. Oceanogr. 35, 826-834.

13. Jayne, S. R. (2009). The impact of abyssal mixing parameterizations in an ocean general circulation model. J. Phys. Oceanogr. 39, 1756-1775.

14. Henyey, F. S., Wright, J. \& Flatté, S. M. (1986). Energy and action flow through the internal wave field. J. Geophys. Res. 91, 8487-8495.

15. Gregg, M. C., Sanford, T. B. \& Winkel, D. P. (2003). Reduced mixing from the breaking of internal waves in equatorial waters. Nature 422, 513-515.

16. Harrison, M. \& Hallberg, R. (2008). Pacific subtropical cell response to reduced equatorial dissipation. J. Phys. Oceanogr. 38, 1894-1912.

17. Lien, R.-C., D'Asaro, E. A. \& McPhaden, M. (2002). Internal waves and turbulence in the Upper Centeral Equatorial Pacific: Lagrangian and eulerian observations. J. Phys. Oceanogr. 32, 2619-2639.

18. Wunsch, C. \& Ferrari, R. (2004). Vertical mixing, energy and the general circulation of the oceans. Ann. Rev. Fluid Mech. 36, 281-412.

19. St. Laurent, L. \& Garrett, C. (2002). The role of internal tides in mixing the deep ocean. J. Phys. Oceanogr. 32, 2882-2899.

20. Alford, M. H. (2001). Internal swell generation: The spatial distribution of energy flux from the wind to mixed layer near-inertial motions. J. Phys. Oceanogr. 31, 2359-2368.

21. D'Asaro, E. (1985). The energy flux from the wind to nearinertial motions in the mixed layer. J. Phys. Oceanogr. 15, 943-959.

22. D'Asaro, E. et al. (1995). Upper-ocean inertial currents forced by a strong storm. Part 1: Data and comparisons with linear theory. J. Phys. Oceanogr. 25, 2909-2936.

23. Alford, M. H. (2003). Energy available for ocean mixing redistributed though long-range propagation of internal waves. Nature 423, 159-163.

24. Polzin, K. L., Toole, J. M. \& Schmitt, R. W. (1995). Finescale parameterizations of turbulent dissipation. J. Phys. Oceanogr. 25, 306-328.

25. Osborn, T. R. (1980). Estimates of the local rate of vertical diffusion from dissipation measurements. J. Phys. Oceanogr. 10, 83-89.

26. Oakey, N. S. (1982). Determination of the rate of dissipation of turbulent energy from simultaneous temperature and velocity shear microstructure measurements. J. Phys. Oceanogr. 12, 256-271.

27. Thorpe, S. (1977). Turbulence and mixing in a Scottish Loch. Philos. Trans. R. Soc. London Ser. A 286, 125-181.
28. Dillon, T. M. (1982). Vertical overturns: A comparison of Thorpe and Ozmidov length scales. J. Geophys. Res. 87, 9601-9613.

29. Ferron, B., Mercier, H., Speer, K., Gargett, A. \& Polzin, K. (1998). Mixing in the Romanche Fracture Zone. J. Phys. Oceanogr. 28, 1929-1945.

30. Seim, H. E. \& Gregg, M. C. (1994). Detailed observations of a naturally occuring shear instability. J. Geophys. Res. 99, 10049-10073.

31. Klymak, J. M., Pinkel, R. \& Rainville, L. (2008). Direct breaking of the internal tide near topography: Kaena Ridge, Hawaii. J. Phys. Oceanogr. 38, 380-399.

32. Klymak, J. M. et al. (2006). An estimate of tidal energy lost to turbulence at the hawaiian ridge an estimate of tidal energy lost to turbulence at the hawaiian ridge an estimate of tidal energy lost to turbulence at the Hawaiian Ridge. J. Phys. Oceanogr. 36, 1148-1164.

33. Müller, P., Holloway, G., Henyey, F. \& Pomphrey, N. (1986). Nonlinear interactions among internal gravity waves. Rev. Geophys 24, 493-536.

34. Sun, H. \& Kunze, E. (1999). Internal wave/wave interactions: Part II. spectral energy transfer and turbulence production rates. J. Phys. Oceanogr. 29, 2905-2919.

35. Gregg, M. C. (1989). Scaling turbulent dissipation in the thermocline. J. Geophys. Res. 94, 9686-9698.

36. Winters, K. B. \& D'Asaro, E. A. (1997). Direct simulation of internal wave energy transfer. J. Phys. Oceanogr. 27, 19371945.

37. Wijesekera, H., Padman, L., Dillon, T., Levine, M. \& Paulson, C. (1993). The application of internal-wave dissipation models to a region of strong mixing. J. Phys. Oceanogr. 23, 269-286.

38. MacKinnon, J. \& Gregg, M. (2003). Mixing on the latesummer New England shelf - solibores, shear and stratification. JPO 33, 1476-1492.

39. Carter, G. S., Gregg, M. \& Lien, R.-C. (2005). Internal waves, solitary waves, and mixing on Monterey Bay shelf. Continental Shelf Research 25, 1499-1520.

40. Mauritzen, C., Polzin, K. L., McCartney, M. S., Millard, R. C. \& West-Mack, D. E. (2002). Evidence in hydrography and density fine structure for enhanced vertical mixing over the mid-atlantic ridge in the western Atlantic. J. Geophys. Res. 107, doi:10.1029/2001JC001114.

41. Sloyan, B. M. (2005). Spatial variability of mixing in the Southern Ocean. Geophys. Res. Lett. 32, L18603, doi:10.1029/2005GL023568.

42. Walter, M., Mertens, C. \& Rhein, M. (2005). Mixing estimates from a large-scale hydrographic survey in the North Atlantic. Geophys. Res. Lett. 32, doi:10.1029/2005GL022471.

43. Stober, U., Walter, M., Mertens, C. \& Rhein, M. (2008). Mixing estimates from hydrographic measurements in the deep western boundary current of the North Atlantic. Deep Sea Research Part I: Oceanographic Research Papers 55, 721 736.

44. Naveira Garabato, A., Polzin, K., King, B., Heywood, K. \& Visbeck, M. (2004). Widespread intense turbulent mixing in the Southern Ocean. Science 303, 210-213. 
45. Galbraith, P. \& Kelley, D. (1996). Identifying overturns in CTD profiles. J. Atmos. Ocean. Tech. 13, 688-702.

46. Gargett, A. \& Garner, T. (2008). Determining Thorpe scales from ship-lowered CTD density profiles. J. Atmos. Ocean. Tech. 25, 1657-1670.

47. Thompson, A. F., Gille, S. T., MacKinnon, J. A. \& Sprintall, J. (2007). Spatial and temporal patterns of small-scale mixing in Drake Passage. J. Phys. Oceanogr. 37, 572-592.

48. Sanford, T. B., Price, J. F., Girton, J. B. \& Webb, D. C. (2007). Highly resolved observations and simulations of the ocean response to a hurricane. Geophys. Res. Lett 34, L13604, doi:10.1029/2007GL029679.

49. Sanford, T. B., Price, J. F. \& Girton, J. B. (2011). Upper ocean response to Hurricane Frances (2004) observed by profiling EM-APEX floats. J. Phys. Oceanogr. in press.

50. Chaigneau, A., Pizarro, O. \& Rojas, W. (2008). Global climatology of near-inertial current characteristics from Lagrangian observations. Geophys. Res. Lett 35, L13603. Doi:10.1029/2008GL034060.
51. Lumpkin, R. \& Pazos, M. Measuring surface currents with SVP drifters: the instrument, its data, and some recent results. In Griffa, A., Kirwan, A. D., Mariano, A., Ozgokmen, T. \& Rossby, T. (eds.) Lagrangian Analysis and Prediction of Coastal and Ocean Dynamics, 39-67 (Cambridge University Press, 2007).

52. Elipot, S. \& Lumpkin, R. (2008). Spectral description of oceanic near-surface variability. Geophys. Res. Lett 35, L05606. Doi:10.1029/2007GL032874.

53. Park, J. J., Kim, K. \& King, B. A. (2005). Global statistics of inertial motions. Geophys. Res. Lett. 32, L14612.

54. Elipot, S., Lumpkin, R. \& Prieto, G. (2010). Intertial oscillations modification by mesoscale vorticity. J. Geophys. Res. 114, doi:10.1029/2008JC005170.

55. Pollard, R. T. \& Millard, R. C. J. (1970). Comparison between observed and simulated wind-generated inertial oscillations. Deep-Sea Res. 17, 813-821. 